\title{
Perfil bibliométrico da produção científica (inter)nacional da Enfermagem Gerontogeriátrica
}

\author{
Bibliometric profile the (inter)national scientific production of Geriatric Nursing
}

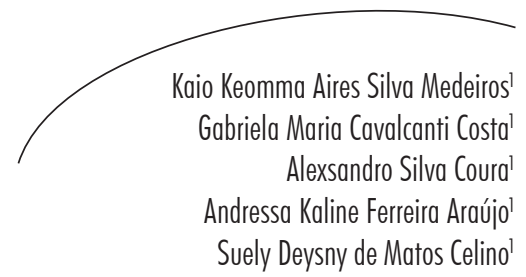

Resumo

Objetivo: Traçar o perfil bibliométrico da produção científica nacional e internacional da Enfermagem Gerontogeriátrica. Método: Estudo bibliométrico realizado nas bases de dados BDENF, IBECS, LILACS, MEDLINE, PubMed e SciELO, utilizando os descritores: enfermagem, assistência de enfermagem, idoso, saúde do idoso e geriatria. Foram selecionados 324 manuscritos, que foram gerenciados no programa JabRef Reference Manager 2.5, e os dados provenientes foram submetidos a análise estatística no software SPSS. Resultados: Verificou-se que a publicação entre 2002 e 2011 é mais extensa; a maioria dos autores é formada por mestres ou doutores; as referências nacionais apresentam maior frequência; os artigos selecionados são, em sua maioria, de natureza quantitativa, de corte transversal e versam sobretudo acerca de doenças crônicas não transmissíveis; há carência de estudos em temáticas como: ética, acessibilidade e participação social; a maioria desses estudos foi classificada nos níveis de evidência 4 e 5, e publicados, em grande parte, nos periódicos com Qualis A1, A2 e B2. Conclusão: A produção científica da Enfermagem Gerontogeriátrica apresenta crescimento. Entretanto, existe a necessidade de os enfermeiros desenvolverem estudos considerando mais a literatura internacional, a interdisciplinaridade e os aspectos relacionados a independência e inserção social e de saúde do idoso.

\section{Abstract}

Objective: Describe the bibliometric profile of the national and international scientific production of Geriatric Nursing. Method: Bibliometric study in BDENF, IBECS, LILACS, MEDLINE, PubMed, and SciELO databases, using the descriptors: nursing, nursing care, elderly, elderly health and geriatrics. We selected 324 manuscripts, which were managed in the program JabRef Reference Manager 2.5, and the data was submitted to statistical analysis using the SPSS software. Results: Publication from 2002 to 2011 is more extensive; most writers are masters or doctors; national references are more frequent; the selected articles are mostly quantitative, cross-sectional and deal with chronic diseases mainly; there is a lack of studies on subjects such as ethics,

Palavras-chave:

Enfermagem. Enfermagem Geriátrica. Saúde do Idoso. Revisão.
Key words: Nursing. Geriatric Nursing. Health of the Elderly. Review. 
accessibility and social participation. Most of these studies were classified into evidence levels 4 and 5, and largely published in Qualis A1, A2 and B2 journals. Conclusion: The scientific nursing production on Geriatrics is growing, but there is a need for nurses to develop research considering the international literature, and interdisciplinary issues related to independence, social inclusion and health of the elderly.

\section{INTRODUÇÃO}

O aumento da proporção de idosos na sociedade é relativamente recente. ${ }^{1}$ A literatura científica revela uma projeção crescente e notória desses indivíduos que, de acordo com alguns organismos internacionais, é verificada em nível mundial. ${ }^{2-3}$

No Brasil, entre os anos de 1997 e 2007, a população de um modo geral apresentou crescimento relativo da ordem de $21,6 \%$, ao passo que a de idosos mais que dobrou, com aumento de aproximadamente $47,8 \%{ }^{4}$ Nesse contexto, acredita-se que o processo de envelhecimento populacional brasileiro assume características próprias, sobretudo em decorrência da velocidade da transição demográfica. ${ }^{5}$

Diante disto, a preocupação com esse novo perfil populacional vem gerando discussões, e a realização de estudos com o objetivo de fornecer dados que subsidiem o desenvolvimento de políticas e programas adequados para os idosos. ${ }^{2}$ A tendência atual desses indivíduos é apresentar mais afecções, gerando, consequentemente, inquietações para a sociedade civil e, em especial, para pesquisadores. ${ }^{6}$ Ademais, o interesse pelo desenvolvimento de pesquisas em Gerontogeriatria se deu também devido à criação das leis específicas para garantir os direitos dos idosos, tais como o Estatuto do Idoso e a Política Nacional de Saúde da Pessoa Idosa, bem como o incentivo para instituir o ensino de Geriatria e Gerontologia nas universidades?

Nessa perspectiva, e entendendo ser a atividade de pesquisa fundamental para o avanço do conhecimento, ${ }^{8}$ o idoso tem sido cada vez mais focalizado nos trabalhos científicos, com variadas abordagens e em diversas áreas do conhecimento, em especial aquelas pertencentes às Ciências da Saúde, a exemplo da Enfermagem Gerontogeriátrica. Não obstante, apesar de ser relevante a produtividade neste campo do conhecimento, verificam-se lacunas relacionadas ao aspecto qualitativo, ${ }^{9}$ ou seja, a efetiva contribuição dos estudos à ciência.

Nesse sentido, acredita-se na pertinência do presente estudo, dadas as circunstâncias atuais de crescimento acelerado na população idosa e constante alta na prevalência de doenças crônicas não transmissíveis. Os aspectos relativos ao idoso e ao envelhecimento se tornam temáticas prementes a serem abordadas nos trabalhos dos enfermeiros, sendo inclusive recomendadas no escopo da Agenda Nacional de Prioridades de Pesquisa em Saúde do Brasil. ${ }^{10}$

Destarte, a apreensão de características acerca da referida produção científica favorecerá uma visão particular do que está sendo difundido na comunidade acadêmica, gerando uma implicação prática, na medida em que oferece subsídios para indicar lacunas, avanços e potencialidades.

Frente aos desafios que hão de vir, e também aqueles superados no que concerne à consolidação da Gerontogeriatria como área do conhecimento da Enfermagem, objetivou-se traçar o perfil bibliométrico da produção científica nacional e internacional da Enfermagem Gerontogeriátrica.

\section{MÉTODO}

No sentido de viabilizar o alcance do objetivo proposto, optou-se por realizar um estudo bibliométrico cuja finalidade é reunir e 
sintetizar resultados de pesquisas sobre um tema delimitado ou questão, de maneira sistemática e ordenada, contribuindo para o aprofundamento no conhecimento do tema investigado. ${ }^{11}$

A coleta dos dados foi feita nos meses de maio e julho de 2011. A pesquisa foi iniciada com busca livre nas bases de dados, e considerados todos os resultados obtidos até 2010. Foram escolhidas as bases de dados comumente utilizadas em pesquisas em Ciências da Saúde, a saber: BDENF (Base de Dados da Enfermagem), CidSaúde (Literatura sobre Cidades/Municípios Saudáveis), IBECS (Índice Bibliográfico Espanhol em Ciências da Saúde), LILACS (Literatura Latinoamericana e do Caribe em Ciências da Saúde), MedCarib (Literatura do Caribe em Ciências da Saúde), MEDLINE (Literatura Internacional em Ciências da Saúde), PubMed (Publicações Médicas) e SciELO (Biblioteca Científica Eletrônica On-line).

Foram definidos os descritores: enfermagem; assistência de enfermagem; idoso; saúde do idoso e geriatria. Estes foram agrupados em uma expressão única de busca (Enferm\$ OR Assistência de Enferm\$) AND (Idos\$ OR Saúde do idos\$ OR Geriatr\$), com exceção da BDENF, que representa uma base de dados própria da Enfermagem, tornando-se, portanto, redundante a aplicação do primeiro conjunto. Nesse sentido, a expressão de uso para essa base de dados específica foi: (Idos\$ OR Saúde do idos\$ OR Geriatr\$).

Após a inserção das expressões de busca nas bases de dados, consideraram-se limites de busca: publicações nos moldes de artigos científicos; estudos que possuíam sujeitos de pesquisa com idade igual ou superior a 60 anos e, por último, aqueles disponibilizados de forma gratuita e na íntegra na internet.

Inicialmente, quando da aplicação das expressões de busca nas bases de dados, foram identificados 4.487.175 artigos. Após considerar os limites de busca mencionados, o número foi reduzido a 226.955, representando, portanto, a população de estudos pré-selecionados. Devido ao volume considerável de manuscritos, estimouse o tamanho da amostra, sendo obtido um $n$ probabilístico de 324 artigos. Para estimar tal amostra, utilizou-se a fórmula " $\mathrm{n}=\mathrm{N} \cdot \mathrm{Z}^{2} \cdot \mathrm{P}(1-\mathrm{P}) /$ $(\mathrm{N}-1) \cdot \mathrm{e}^{2}+\mathrm{Z}^{2} \cdot \mathrm{P}(1-\mathrm{P})$ ", em que: $\mathrm{n}=$ valor $\mathrm{da}$ amostra; $\mathrm{N}=$ valor da população; $\mathrm{Z}=$ intervalo de confiança $(1,96) ; \mathrm{P}=$ prevalência; $\mathrm{e}=$ erro tolerado $(0,05)$.

Em seguida, conduziu-se uma amostragem aleatória simples, viabilizada por meio de sorteio efetuado no programa Microsoft Office Excel 2007, de forma proporcional ao número de estudos encontrados em cada base, da seguinte maneira: BDENF ( $n=181)$; IBECS $(n=1)$; LILACS $(n=5)$; MEDLINE ( $\mathrm{n}=34)$; PubMed ( $\mathrm{n}=94)$ e SciELO $(n=9)$. As bases de dados CidSaúde e MedCarib não foram contempladas, por terem apresentado resultados próximos a zero durante os testes de proporcionalidade.

Os artigos sorteados em cada base de dados foram incluídos na amostra quando se adequaram ao critério de inclusão: ser produção da Enfermagem sobre saúde do idoso, verificado pela presença de, no mínimo, um enfermeiro no corpo de autores. Em caso de desconformidade com tal critério, considerou-se o artigo seguinte. Caso também não se enquadrasse, analisouse $\mathrm{o}$ artigo anterior, e assim por diante ( $\mathrm{x} ; \mathrm{x}+1$; $\mathrm{x}-1 ; \mathrm{x}+2 ; \mathrm{x}-2 \ldots$.$) . Para tanto, fez-se uma leitura$ dos títulos e resumos dos mesmos. Cabe ressaltar que as bases de dados utilizadas podem apresentar algumas subdivisões inseridas na interface de outras bases; assim, para minimizar possíveis distorções, os artigos repetidos foram considerados apenas uma vez.

Em sendo condizentes com tais critérios, os estudos foram armazenados com o auxílio do software de gerenciamento de referências JabRef Reference Manager versão 2.5, o qual foi utilizado para gerar fichamentos eletrônicos de cada artigo.

Os dados foram coletados por dois revisores, por meio do preenchimento de um formulário que continha as variáveis bibliométricas a serem 
obtidas com base na análise dos artigos incluídos na amostra, sendo as informações apresentadas em tabelas, gráficos e figuras. Após a coleta, os dados foram inseridos no software Statistical Package for the Social Sciences (SPSS), 17.0, sendo submetidos à estatística descritiva (frequências absolutas e relativas) e ao teste $\mathrm{U}$ de MannWhitney, para permitir comparação das médias de variáveis independentes: ano de publicação e origem das referências.

Para categorização dos artigos de acordo com os estratos do qualis/CAPES, considerou-se a avaliação até o ano de 2010. Para classificação quanto ao poder de evidência, utilizou-se a Classificação Norte-americana Hierárquica de Evidências: nível 1 - metanálise de múltiplos estudos controlados; nível 2 - estudo individual com delineamento experimental; nível 3 - estudo com delineamento quase-experimental como estudo sem randomização com grupo único pré e pós-teste, séries temporais ou caso-controle; nível
4 - estudo com delineamento não-experimental como pesquisa descritiva correlacional e qualitativa ou estudos de caso; nível 5 - relatório de casos ou dado obtido de forma sistemática, de qualidade verificável ou dados de avaliação de programas; nível 6 - opinião de autoridades respeitáveis baseada na competência clínica ou opinião de comitês de especialistas, incluindo interpretações das informações não baseadas em pesquisas. ${ }^{12}$

\section{RESULTADOS}

Na figura 1 está representado o fluxograma de seleção dos artigos. Verifica-se que foram identificados, inicialmente, no momento da aplicação da expressão de busca nas bases de dados, 4.487.175 manuscritos, restando 226.955 após a consideração dos limites de busca e, por fim, após o cálculo amostral, os 324 que compuseram a amostra, distribuídos de forma proporcional nas bases de dados.

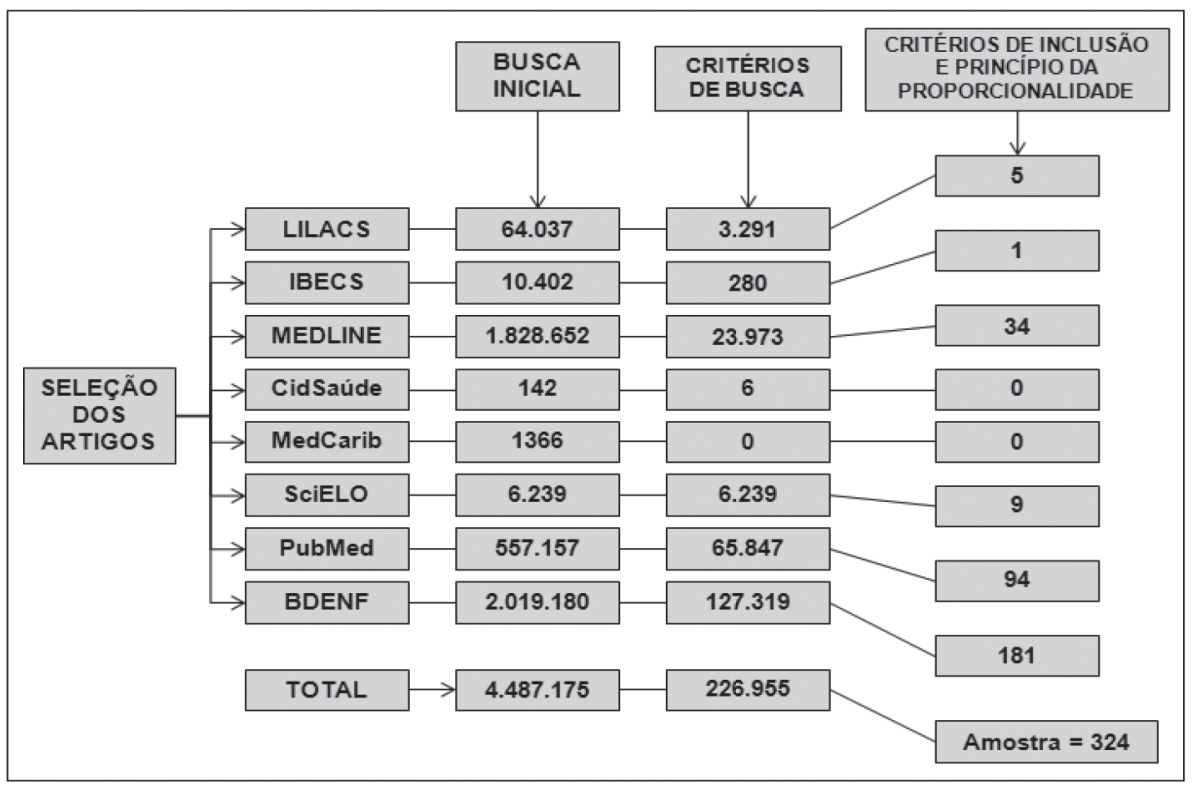

Figura 1. Fluxograma da seleção dos artigos. 
$\mathrm{Na}$ figura 2, pode-se visualizar a evolução temporal do quantitativo de artigos por ano de publicação. Verifica-se uma distribuição crescente e que $82,7 \%$ ( $n=263)$ dos artigos foram publicados nos últimos dez anos; portanto, a publicação de 2002 a 2011 é mais extensa $(p<0,001)$.

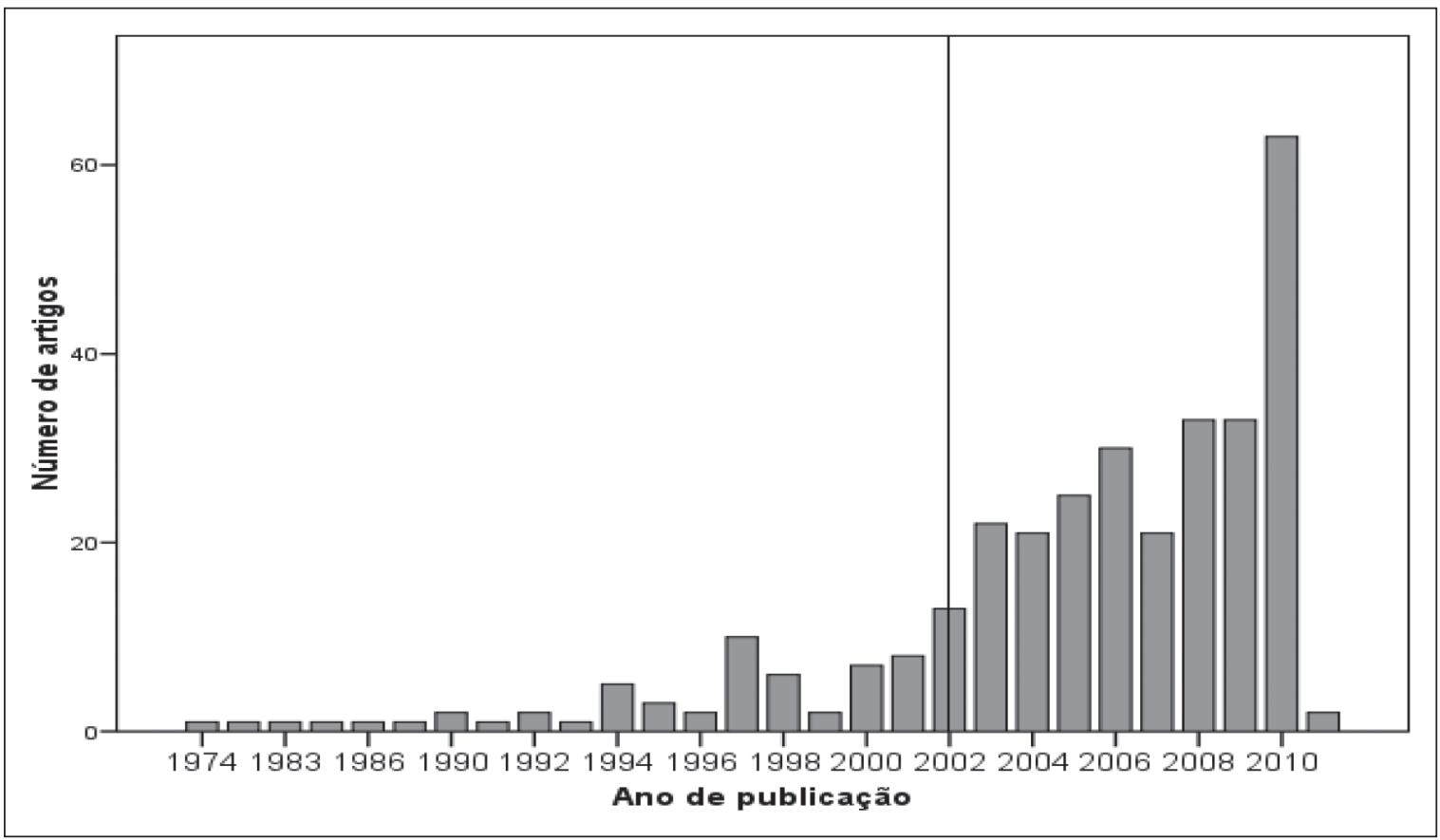

Figura 2. Distribuição dos artigos por ano de publicação.

Apesar de considerar como um dos critérios de elegibilidade, estudos que continham no mínimo um enfermeiro no corpo de autores, alguns artigos possuíam mais de um autor que poderia ser ou não de outras áreas do conhecimento. Isso explica, portanto, a ocorrência de pesquisadores com várias formações profissionais, sendo que quase metade do total de autores dos artigos que compuseram a amostra estudada são enfermeiros (tabela 1). Porém, desconsiderando os autores sem identificação de formação acadêmica, o percentual de enfermeiros atinge $87 \%(\mathrm{n}=367)$.

No concernente à titulação, mais da metade dos autores são mestres ou doutores $(\mathrm{n}=530$ $69 \%)$. Vale salientar, ainda, que $11,4 \%(n=87)$ dos autores são alunos de graduação e pós-graduação. 
Tabela 1. Distribuição dos autores dos artigos selecionados por formação acadêmica e titulação no momento da publicação (n=768). 2011.

\begin{tabular}{|c|c|c|}
\hline Variáveis & $\mathrm{n}$ & $\%$ \\
\hline \multicolumn{3}{|l|}{ Formação acadêmica } \\
\hline Enfermeiro & 367 & 47,8 \\
\hline Informação ausente & 346 & 45 \\
\hline Médico & 19 & 2,5 \\
\hline Fisioterapeuta & 12 & 1,5 \\
\hline Farmacêutico & 6 & 0,8 \\
\hline Psicólogo & 4 & 0,5 \\
\hline Advogado & 3 & 0,4 \\
\hline Sociólogo & 2 & 0,3 \\
\hline Assistente social & 2 & 0,3 \\
\hline Estatístico & 2 & 0,3 \\
\hline Nutricionista & 2 & 0,3 \\
\hline Fonoaudiólogo & 1 & 0,1 \\
\hline Terapeuta ocupacional & 1 & 0,1 \\
\hline Cirurgião-dentista & 1 & 0,1 \\
\hline \multicolumn{3}{|l|}{ Titulação } \\
\hline Doutor & 335 & 43,6 \\
\hline Mestre & 195 & 25,4 \\
\hline Informação ausente & 133 & 17,3 \\
\hline Mestrando & 41 & 5,4 \\
\hline Doutorando & 34 & 4,4 \\
\hline Graduando & 10 & 1,3 \\
\hline Especialista & 8 & 1 \\
\hline Graduado & 7 & 0,9 \\
\hline Pós-doutor & 3 & 0,4 \\
\hline Pós-doutorando & 2 & 0,3 \\
\hline
\end{tabular}


No tocante ao Qualis/Capes dos periódicos nos quais os artigos selecionados foram publicados, identificaram-se: A2 - 39,5\% $(\mathrm{n}=128) ; \mathrm{B} 1$ - 15,7\% ( $\mathrm{n}=51) ; \mathrm{A} 1$ - 3,1\% ( $\mathrm{n}=10) ; \mathrm{B} 2$ - 1,2\% (n=4); e B3 - 0,3\% ( $\mathrm{n}=1)$. Porém, 40,1\% $(\mathrm{n}=130)$ dos periódicos não apresentavam Qualis catalogado na listagem oficial da CAPES.

Com relação ao poder de evidência dos manuscritos selecionados, identificou-se que 90,5\% ( $\mathrm{n}=293$ ) estão entre os níveis 4 ou 5. Em seguida, aparecem: nível 6 (4,9\%), nível 3 (4,3\%) e nível $1(0,3 \%)$.

$\mathrm{Na}$ figura 3, a avaliação das 8.241 referências bibliográficas evidenciou uma média de 25,43 referências por artigo, bem como, de modo geral, crescimento progressivo das mesmas ao longo dos anos. Verificou-se que a maioria das referências é nacional $(\mathrm{n}=6.463-\mathrm{p}<0,001)$, embora sua tendência seja decrescente, enquanto as internacionais tendem a ser mais citadas.

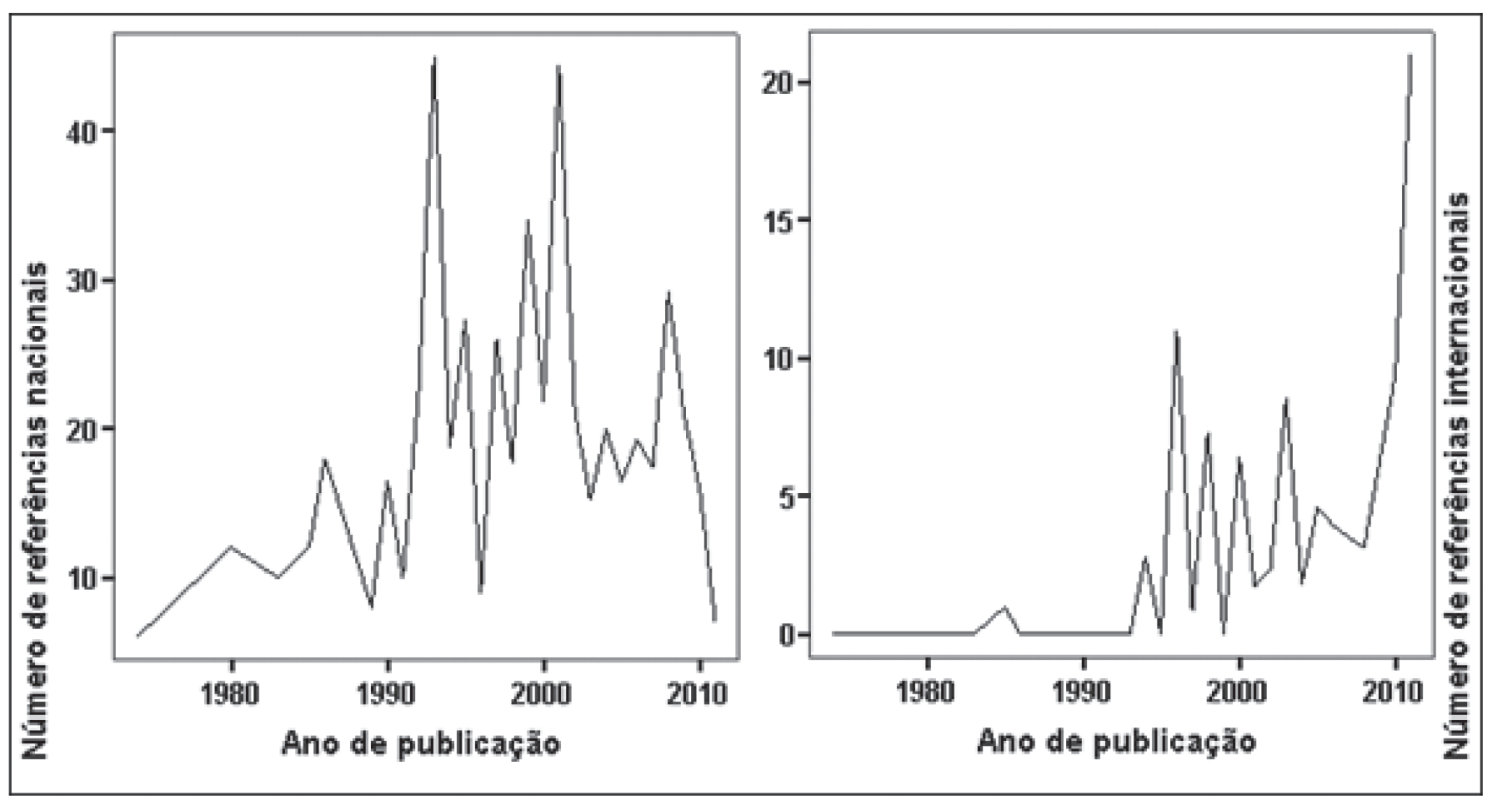

Figura 3. Distribuição das referências por ano de publicação.

Dentre as referências utilizadas nos artigos selecionados, identificou-se que 5.655 são oriundas de revistas. Dentre elas, 841 são especializadas em Enfermagem e 4.814 são de outras áreas. Identificaram-se ainda 1.782 citações de livros, 107 de dissertações de mestrado, 96 de teses de doutorado, 366 de documentos institucionais e 227 de outras fontes.
Na tabela 2, podem-se visualizar os conteúdos ou enfoques principais dos artigos. A maior frequência foi de manuscritos que tinham as doenças crônicas não transmissíveis (DCNT), a qualidade de vida, os cuidadores ou a capacidade funcional como objetos de estudo. Os temas menos enfocados foram: ética, acessibilidade, saúde bucal e participação social. 
Tabela 2. Distribuição dos conteúdos das publicações segundo os enfoques principais. 2011.

\begin{tabular}{|c|c|c|}
\hline Temas & $\mathrm{n}$ & $\%$ \\
\hline Doenças crônicas não transmissíveis & 73 & 22,5 \\
\hline Qualidade de vida & 43 & 13,3 \\
\hline Cuidadores & 31 & 9,6 \\
\hline Capacidade funcional & 29 & 9,1 \\
\hline Saúde mental & 24 & 7,4 \\
\hline Enfermagem clínica & 24 & 7,4 \\
\hline Sistematização da assistência & 19 & 5,8 \\
\hline Atenção primária & 18 & 5,5 \\
\hline Agravos externos/quedas & 17 & 5,2 \\
\hline Ações de autocuidado & 13 & 4,1 \\
\hline Políticas públicas & 10 & 3,1 \\
\hline Tecnologias & 7 & 2,2 \\
\hline Nutrição & 4 & 1,2 \\
\hline Discussão de gênero & 3 & 0,9 \\
\hline Educação e comunicação & 3 & 0,9 \\
\hline Drogas & 2 & 0,6 \\
\hline Ética e bioética & 1 & 0,3 \\
\hline Acesso aos serviços de saúde & 1 & 0,3 \\
\hline Saúde bucal & 1 & 0,3 \\
\hline Participação social & 1 & 0,3 \\
\hline Total & 324 & 100 \\
\hline
\end{tabular}

No tocante à temporalidade de acompanhamento da amostra, 79,3\% ( $\mathrm{n}=257)$ dos artigos são transversais, em detrimento dos $20,7 \% \quad(n=67)$ de artigos com desenho longitudinal.

Verificou-se que os estudos quantitativos foram mais frequentes do que os artigos com abordagem qualitativa, gerando uma proporção de 1,6/1. Evidenciou-se que trabalhos com natureza metodológica mista foram mais raros, representando 4,3\% da amostra.

\section{DISCUSSÃO}

O considerável aumento na longevidade populacional, verificado em muitos países, mpacta sobremaneira os indicadores epidemiológicos. Esses fatores ocasionam entraves ao sistema de saúde em vários países e, consequentemente, preocupações para entidades de pesquisa que buscam minimizar tal problemática.

A Enfermagem Gerontogeriátrica vem assumindo importantes atribuições no cuidado 
do idoso e um privilegiado lugar no âmbito das Ciências da Saúde, na medida em que busca agrupar fundamentos da enfermagem geral e conhecimentos próprios da Geriatria e da Gerontologia. Todavia, para que se alcance o desenvolvimento desta área, o caminho da pesquisa é inevitável. ${ }^{13}$

O elevado quantitativo de estudos distribuídos nas bases de dados apresentado na figura 1 é indicativo de que o crescimento da população de idosos pode ter impacto em diversos setores, dentre os quais se destaca a produção do conhecimento. Ao que parece, isso demonstra a importância que a temática vem tendo no âmbito acadêmico e científico.

Salienta-se, pois, que apesar de o considerável aumento das populações idosas em diversos países contribuir para o crescimento dos estudos em Geriatria e Gerontologia, deve-se creditar tal acontecimento também ao boom das ciências de uma forma geral, das bases de dados (aumento do número de revistas e edições), das possibilidades de financiamento de estudos, bem como do surgimento de políticas públicas voltadas para o idoso.

Outrossim, a popularização da internet como ferramenta eficiente na disseminação e popularização de conhecimentos possui grande influência no aumento das publicações da Enfermagem Gerontogeriátrica. A estratégia da Biblioteca Virtual em Saúde (BVS), por exemplo, tem colaborado com a reestruturação do fluxo da comunicação científica, objetivando promover a convergência dos diferentes atores, num único espaço virtual de domínio público. ${ }^{14}$ Apesar disso, há de se considerar que algumas revistas de Enfermagem, sobretudo as brasileiras, foram inseridas em determinadas bases de dados on-line há poucos anos. Assim sendo, há volumes dessas revistas, a exemplo da Revista da Escola de Enfermagem da USP, que só possuem edições indexadas na SciELO a partir de 1996.

Em estudo que objetivou avaliar a construção histórica do conhecimento da Enfermagem em
Gerontogeriatria no Brasil, verificou-se que seu quantitativo começou a ser expressivo em meados de 1985, mas se manteve pequeno no decorrer da década. ${ }^{7}$ Em contrapartida, observando o figura 2, percebe-se o aumento das publicações da Enfermagem na área no início do século XXI, quando o número de artigos ultrapassou todos aqueles publicados anteriormente.

Tal aumento também coincide com a implementação da Política Nacional de Ciência, Tecnologia e Inovação em Saúde, aprovada em 2004, ${ }^{15}$ juntamente com a Agenda Nacional de Prioridades de Pesquisa em Saúde ${ }^{10}$ quando houve mudança no panorama da pesquisa em saúde no país, diante da urgência de estimular estudos voltados às necessidades de saúde da população brasileira. ${ }^{15}$ Esse crescimento culminou com a motivação dos pesquisadores da área em buscar conhecimentos com enfoque no idoso, desenvolvendo pesquisas que retratassem aspectos relacionados às alterações físicas e sociais inerentes ao envelhecimento, ${ }^{16}$ por exemplo.

Por outro lado, pensar no quantitativo de publicações não revela a resolutividade dos problemas advindos da longevidade populacional. Apesar dos avanços nas pesquisas no que se refere, principalmente, ao tratamento de DCNT, os sistemas nacionais de saúde enfrentam dificuldades para tornar estes novos tratamentos acessíveis à maioria das pessoas. ${ }^{17}$ Ademais, em estudo recente, cujo público-alvo foi representado, em parte, por pesquisadores, em torno de $75 \%$ dos entrevistados consideraram que existem tópicos de pesquisa ainda negligenciados no Brasil, tais como aqueles relativos ao envelhecimento. ${ }^{18}$

Entretanto, compreende-se que ocorreram avanços no tocante, por exemplo, à interdisciplinaridade na produção do conhecimento gerontogeriátrico, contribuindo sobremaneira para a formação dos acadêmicos de Enfermagem e possibilitando o intercâmbio de conhecimento entre os profissionais da área de saúde. ${ }^{13}$ A esse respeito a tabela 1 trata, em 
parte, da distribuição dos autores dos artigos selecionados por formação acadêmica. Os dados sugerem a contribuição de outras ciências nas produções selecionadas em conjunto com a Enfermagem, sendo as mais expressivas advindas da Medicina, da Fisioterapia e, em menor escala, da Psicologia.

Contudo, acredita-se que a abordagem interdisciplinar é implementada com restrições, apesar de ser preconizada e verificada em determinadas situações. ${ }^{19}$ Inúmeros fatores concorrem para esta constatação, cabendo citar o ainda recente incentivo da produção do conhecimento na área, bem como o fato de que muitas ciências do campo da saúde não possuem tradição de trabalho em conjunto com a Enfermagem.

Espera-se que em alguns anos esses fatores exerçam pouca influência sobre a produção científica da Enfermagem Gerontogeriátrica, mesmo porque a contribuição da interdisciplinaridade representa uma prática de trabalho científico e profissional benéfica para o idoso, servindo como instrumento condutor para a Gerontogeriatria no seu processo de desenvolvimento. ${ }^{20}$ Além disso, a união e formação de uma rede de colaboração entre autores visa contribuir com a expansão do conhecimento científico, como também almeja a justaposição e articulação de conhecimentos, conceitos e teorias oriundos de diferentes áreas, além da promoção de maior divulgação da pesquisa. ${ }^{21}$

Sem compreender a Gerontogeriatria à luz da interdisciplinaridade, a produção científica da Enfermagem dificilmente conseguirá superar abordagens convencionais e propor novos caminhos para a compreensão de aspectos inerentes ao idoso. As Ciências da Saúde ainda estão no processo de construção do conhecimento gerontogeriátrico e necessitam, portanto, de uma complementaridade para que juntas alcancem a implementação de seus conhecimentos sobre o assunto e publicações de relevância científica.

Analisando a importância do conhecimento produzido pela Enfermagem, refletido na forma e no impacto de sua divulgação, supõese que a mesma tem alcançado publicações em periódicos considerados com satisfatória circulação e abrangência geográfica, visto que quando se analisou o Qualis/Capes, uma espécie de estratificação de qualidade das revistas científicas, os artigos selecionados encontravamse, em sua maioria, nos estratos A1, A2 e B1.

Não obstante, no que se refere ao poder de evidência dos estudos, verificou-se que estes estão centrados majoritariamente nos níveis 4 e 5, em uma escala que vai de 1 a 6 , sendo, portanto, considerados relativamente baixos. Isso se deve, em parte, às características inerentes ao saber gerontogeriátrico, cuja abordagem é fundamentalmente fenomenológica. Ademais, entende-se que a Enfermagem vem buscando mecanismos que visem à sofisticação e aprimoramento de sua produção. Prova disto é que cerca de $70 \%$ dos autores mencionados na tabela supracitada são mestres e/ou doutores. Não é regra, mas isso possibilita agregar credibilidade e relevância ao conhecimento que estes almejam publicar.

Sob outra perspectiva, o considerável número de mestres e/ ou doutores identificados neste estudo está associado, também, ao aumento da oferta de cursos de pós-graduação stricto sensu nas últimas duas décadas, tendo como um dos principais objetivos a formação de pesquisadores capazes de desenvolver pesquisas científicas. ${ }^{22,23}$ No Brasil, a criação da Universidade Aberta do Sistema Único de Saúde (UnA-SUS), em 2008, representou um importante aporte de investimentos para a qualificação desses profissionais, com vistas à criação de uma rede colaborativa de qualificação permanente. ${ }^{24}$

Todavia, a interdependência entre as nações, proporcionada pela globalização, criou sua própria dinâmica e a saúde tem se mostrado um elemento essencial em relações exteriores e assuntos de interesse internacional. ${ }^{25}$ Nesse sentido, além da já referida qualificação profissional, os pesquisadores vêm buscando, cada vez mais, referenciar estudos internacionais em seus trabalhos (figura 3). Acredita-se, pois, 
que uma ação como esta representa um esforço e reconhecimento da necessidade da Enfermagem no sentido de favorecer a internacionalização do conhecimento e o intercâmbio de informações com outros centros de pesquisa. Essa prática propicia um fluxo profícuo entre os países, de saberes e fazeres, que favorece o compartilhamento de ideias e práticas, de modo a ampliar os horizontes e responder às demandas da profissão. ${ }^{26}$

Por outro lado, para além de apontar que a Enfermagem Gerontogeriátrica vem demandando esforços para ainternacionalização do saber, no que se refere particularmente às referências em diversos idiomas, sabe-se que o aumento substancial na quantidade de estudos desenvolvidos na área em questão fez com que as revistas implementem procedimentos restritivos para selecionar os que possuem potencial para publicação, como a consideração da literatura internacional pelos pesquisadores, haja vista que há entendimento de que os assuntos relativos à saúde não pode mais permanecer puramente nacional.

Assim como os motivos que levaram a Enfermagem Gerontogeriátrica - e a ciência em geral - a trabalhar na perspectiva da internacionalização do saber-fazer, as dificuldades para mobilizar esse processo incluem aspectos de diferentes naturezas, mas concentram-se numa causa comum, qual seja, tornar as publicações mais atrativas para os autores e editores de periódicos estrangeiros.

Experts da enfermagem brasileira foram convidados a discorrer sobre o tema em questão e apontaram que os obstáculos para tornar o conhecimento, em especial o produzido no Brasil, como algo internacional, variam desde a seleção de temas que estejam vinculados a demandas contemporâneas e integrados à profissão, para além de seus limites geográficos, passando pela realização de estudos com delineamentos metodológicos de rigor inquestionável e que ultrapassem a fase diagnóstica, até a restrição em ler e escrever na língua inglesa. ${ }^{26}$
Ante as dificuldades retromencionadas, é premente o estabelecimento de estratégias que superem essa realidade e, como não podia ser diferente, abrangem a mobilização dos pesquisadores da Enfermagem Gerontogeriátrica em diversos aspectos. Dentre tais aspectos se enumeram, prioritariamente, estímulo à realização de intercâmbios de estudantes e pesquisadores, desde a graduação e em especial após o doutoramento, para vários centros internacionais, bem como investir no conhecimento de línguas, em especial o idioma inglês, empenhandose na publicação de artigos neste idioma. Uma publicação acerca do tema acrescenta a necessidade de aprofundar a análise e discussão dos resultados de pesquisas, assim como explicitar as implicações destes resultados nas diferentes conjunturas, de modo que nossos produtos sejam mais atrativos para inserção internacional, o que não implica falta de sensibilidade social para problemas que nos são peculiares. ${ }^{26}$

Apesar dos esforços da Enfermagem no sentido de agregar maior cientificidade a suas publicações, percebe-se ainda o predomínio da abordagem transversal, quanto à temporalidade de acompanhamento da amostra. Assim, ratificase ainformação acerca do baixo poder de evidência das pesquisas em Gerontogeriatria publicados pela Enfermagem e identificados nesta pesquisa. Tal fato pode ser explicado, pois estudos transversais não requerem muita infraestrutura, custos e tempo quando comparados aos que possuem abordagem longitudinal. No entanto, também são importantes, pois permitem caracterizar vários aspectos do processo saúdedoença como prevalências, associações entre as variáveis e formulação de hipóteses. ${ }^{27}$

No que se refere, ainda, a questões metodológicas, a pesquisa constatou estudos em sua maioria de cunho quantitativo, mesmo com a atual tendência de investigações com abordagens qualitativas na área da Enfermagem, verificada a partir da década de 1980. Esta demarcou o início de uma transição na perspectiva de investigação científica antipositivista, a qual permitia uma visão parcial dos processos vivenciais. ${ }^{27}$ 
Este é um fator preocupante, pois os idosos vivenciam uma mudança de paradigmas, resultante de transformações nos âmbitos social, econômico e cultural, acarretando a necessidade de novos olhares e planejamentos, para contemplar suas expectativas de saúde e atender às demandas biopsicossociais visando ao equilíbrio do processo vital. ${ }^{16}$ Tais características são típicas, do ponto de vista metodológico, de pesquisas de cunho qualitativo.

Acredita-se que, devido às próprias limitações inerentes à abordagem quantitativa - por exemplo, não possibilitar ao pesquisador discutir amplamente questões de ordem psicossociocultural -, constatou-se, nesta pesquisa, maior prevalência de estudos sobre DCNT. Isso leva-se ao entendimento de que há maior preocupação à prática curativa, corroborada pela carência de estudos com temáticas como ética, acessibilidade e participação social (tabela 2). Tal assertiva ratifica outra pesquisa, na qual se indica que os profissionais de Enfermagem e ciências afins estão mais preocupados com a investigação sobre situações curativas do processo saúde/doença. ${ }^{16}$

Em virtude do aumento na longevidade populacional, acredita-se que em algumas décadas as DCNTs, comum em idosos, serão ainda mais responsáveis pelas causas de morbimortalidade entre as populações cada dia mais afetadas pelo processo de envelhecimento humano. Iss, em tese, justifica o maior estímulo em pesquisas com enfoque em diagnóstico de doenças, tratamento e reabilitação, tal como supõe estar acontecendo, com base nas observações do presente estudo.

Não obstante, é importante ressaltar que a mudança no perfil demográfico que colocou em ascensão os idosos em todo o mundo, bem como a que vem ocorrendo na esfera epidemiológica, com o gradativo aumento das DCNTs em detrimento da redução substancial das doenças infecto-contagiosas, resulta de transformações de ordem política, econômica e cultural, e não apenas de atributos biológicos.
Isto posto, torna-se premente a reorientação dos estudos na área da Enfermagem Gerontogeriátrica, devendo para tal intento ser contemplado com práticas que ultrapassem o diagnóstico e tratamento de doenças, de forma que o envelhecimento e o idoso sejam compreendidos a partir de uma perspectiva social. Com isso, espera-se alcançar mudanças nas políticas públicas para amparar de forma adequada o idoso, investindo-se principalmente na atenção primária com rediscussões de estratégias preventivas e de promoção à saúde, conforme proposto em outro estudo. ${ }^{16}$

Apesar das informações verificadas nesta pesquisa, compreende-se que a mesma apresenta a limitação de considerar apenas os artigos disponíveis on-line e na íntegra, pois tal procedimento pode evitar a inclusão de artigos com potencial para atender ao objetivo proposto. Além disso, alguns volumes das revistas não estão nas bases de dados.

\section{CONCLUSÃO}

Constata-se que a produção científica da Enfermagem Gerontogeriátrica cresce em ritmo acelerado, decerto na tentativa de atender às demandas do emergente grupo populacional de idosos verificadas nos últimos anos, no Brasil e demais países. Ademais, o boom das ciências de uma forma geral, da internet, das bases de dados (aumento do número de revistas e edições) e das possibilidades de financiamento de estudos, bem como o incremento de políticas públicas focadas no idoso, também figuram como importantes fatores que contribuem para o aumento substancial na produção gerontogeriátrica da Enfermagem.

Mesmo considerando esse aumento, apreendeuse que tal produção ainda apresenta lacunas. Os artigos selecionados, apesar de publicados em importantes periódicos indexados e versarem, principalmente, sobre doenças crônicas não transmissíveis (DCNTs), maior problema que acomete os idosos em todo o mundo hoje, são 
em sua maioria de baixo poder de evidência. Além disso, não se pode pensar a Enfermagem Gerontogeriátrica sob outra perspectiva senão a da interdisciplinaridade, o que favorece o aprimoramento do conhecimento científico e a consequente melhoria da assistência prestada à população idosa numa perspectiva integral. Talvez essa seja uma das maiores lacunas que ainda permeiam os estudos científicos da Enfermagem.

Apesar desses aspectos, vale salientar que a Enfermagem, nos últimos anos, vem

\section{REFERÊNCIAS}

1. Figueiredo D, Lima MP, Sousa L. Os "pacientes esquecidos": satisfação com a vida e percepção de saúde em cuidadores familiares de idosos. Rev Kairós 2009;12(1):97-112.

2. Mendes MRSSB, Gusmão JL, Faro ACM, Leite RCBO. A situação social do idoso no Brasil: uma breve consideração. Acta paul enferm 2005;18(4):422-6.

3. Salgueiro H, Lopes M. A dinâmica da família que coabita e cuida de um idoso dependente. Rev gaúch enferm 2010;31(1):26-32.

4. Instituto Brasileiro de Geografia e Estatística. Síntese de indicadores sociais: uma análise das condições de vida da população brasileira 2008 [Internet]. Rio de Janeiro: IBGE; 2008 [citado 09 Abr. 2012]. (Estudos e Pesquisas). (Informação Demográfica e Socieconômica, 23). 279 p. Disponível em: http://www.ibge.gov.br/home/estatistica/ populacao/condicaodevida/indicadoresminimos/ sinteseindicsociais2008/indic_sociais2008.pdf

5. Carreira L, Rodrigues RAP. Estratégias da família utilizadas no cuidado ao idoso com condição crônica. Ciênc cuid saúde 2006;5(Supl 1):119-26.

6. Alves LC, Leimann BCQ, Vasconcelos MEL, Carvalho MS, Vasconcelos AGG, Fonseca TCO, et al. A influência das doenças crônicas na capacidade funcional dos idosos do município de São Paulo, Brasil. Cad saúde pública 2007;23(8):1924-30.

7. Kletemberg DF, Padilha MI, Gonçalves LHT, Borenstein MS, Alvarez AM, Ferreira AC. A construção histórica do conhecimento da enfermagem gerontológica no Brasil. Esc Anna Nery Rev Enferm 2010;14(4):787-96. demandando esforços para a melhoria de suas publicações. Prova disso é o número considerável de pesquisadores com pós-graduação stricto senso, possibilitando agregar a seus trabalhos credibilidade e relevância científica.

A contribuição que o estudo apresenta é a indicação da necessidade de os enfermeiros desenvolverem pesquisas considerando mais a literatura internacional, a interdisciplinaridade e os aspectos relacionados a independência e inserção social e de saúde do idoso.

8. Teixeira MA, Nitschke RG, Paiva MS. Análise dos dados em pesquisa qualitativa: um olhar para a proposta de Morse e Field. Rev Rene 2008;9(3):125-34.

9. Alvarado, RU. A produtividade dos autores na literatura de enfermagem: um modelo de aplicação da lei de Lotka. Inf \& Soc Est 2006;16(1):63-78.

10. Brasil. Ministério da Saúde, Secretaria de Ciência, Tecnologia e Insumos Estratégicos, Departamento de Ciência e Tecnologia. Agenda Nacional de Prioridades de Pesquisa em Saúde. 2.ed. Brasília: Ministério da Saúde; 2008.

11. Souza MT, Silva MD, Carvalho R. Revisão integrativa: o que é e como fazer? Einstein (São Paulo) [Internet] 2010 [citado 10 abr. 2012];8(1):102-6. Disponível em: http://apps.einstein.br/revista/arquivos/PDF/1134Einsteinv8n1_p102-106_port.pdf

12. Galvão CM. Níveis de Evidência. Acta Paul Enferm [Internet] 2006 [citado 29 Set. 2012];19(2):5.

Disponível em: http://www.scielo.br/pdf/ape/v19n2/ a01v19n2.pdf

13. Hammerschmidt KSA, Borghi ACS, Lenardt $\mathrm{MH}$, Seima MD. Pesquisas de enfermagem em gerontologia. Cogitare enferm 2007;12(2):214-21.

14. Castro RCF. Impacto da Internet no fluxo de comunicação científica em saúde. Rev saúde pública 2006;40(n.esp):57-63.

15. Brasil. Ministério da Saúde, Secretaria de Ciência, Tecnologia e Insumos Estratégicos, Departamento de Ciência e Tecnologia. Política Nacional de Ciência, Tecnologia e Inovação em Saúde. 2.ed. Brasília: Ministério da Saúde; 2006. 
16. Barreira KS, Vieira LJES. O olhar da enfermagem para o idoso: revisão de Literatura. Rev Enferm UERJ 2004;12:332-7.

17. Noronha JC, Silva TR, Fernando S, Barata RB. O que os pesquisadores pensam do sistema de pesquisa em saúde no Brasil: um estudo piloto. RECIIS [Internet] 2012 [citado 29 Set. 2012]; 6(1):12-18. Disponível em: http://www.reciis.cict.fiocruz.br/index.php/reciis/ article/view/478/973

18. Kalache A. O mundo envelhece: é imperativo criar um pacto de solidariedade social. Ciênc saúde coletiva 2008;13(4):1107-11.

19. Motta LB, Aguiar AC. Novas competências profissionais em saúde e o envelhecimento populacional brasileiro: integralidade, interdisciplinaridade e intersetorialidade. Ciênc saúde coletiva 2007;12(2):363-72.

20. Camacho ACLF. A gerontologia e a interdisciplinaridade: aspectos relevantes para a enfermagem. Rev latinoam enferm 2002;10(2):229-33.

21. Marziale MHP. Produção científica da enfermagem brasileira: a busca pelo impacto internacional. Rev latinoam enferm 2005;13(3):285-6.

22. Cruz EA, Alves MDS, Fraga MNO, Damasceno MMC. Abordagem ética em pesquisas publicadas por um programa de pós-graduação em enfermagem. Texto \& contexto enferm 2009;14(1):25-32.
23. Veiga KCG, Menezes TMO. Produção do conhecimento em enfermagem: a (in) visibilidade da atenção à saúde do idoso. Rev Esc Enferm USP 2008;42(4):761-8.

24. Laguardia J. A educação online e os desafios à qualificação profissional em saúde. RECIIS [Internet] 2012 [citado 29 Set. 2012];4(5):40-52. Disponível em: http://www.reciis.cict.fiocruz.br/index.php/reciis/ article/view/393/706

25. Kickbusch I, Berger C. Diplomacia da saúde global. RECIIS [Internet] 2012 [citado 29 Set. 2012];4(1):1924. Disponível em: http://www.reciis.icict.fiocruz. br/index.php/reciis/article/view/342/494 Ver Formatação

26. Barros ALBL, Matheus MCC, Gutiérrez MGR. Internacionalização do saber- fazer da Enfermagem. Acta paul enferm [Internet] 2008 [citado 14 Jul. 2013];21(1):v-v. Disponível em: http://www.scielo. br/scielo.php?script $=$ sci_arttext\&pid=S0103210020 08000100001\&lng=en. http://dx.doi.org/10.1590/ S0103-21002008000100001.

27. Erdmann AL, Silva IA, Rodrigues RAP, Fernandes JD, Vianna LAC, Lopes MJM, et al. Teses produzidas nos programas de pós-graduação em enfermagem de 1983 a 2001. Rev Esc Enferm USP 2005;39(esp):497-505. 